\section{Low dose of fish oil in osteoarthritis: low effect?}

We read with great interest the results of the study from Hill et $a{ }^{1}{ }^{1}$ on fatty acids diet (two regimens of fish oils: low and high daily dose) in patients with painful knee osteoarthritis (OA). As reported in the associated editorial letter from Felson et $a l,{ }^{2}$ this is an impressive work spanning over 2 years, which combines both clinical and MRI data. The authors should be congratulated for the quality of this trial. At the end, there is an unexpected effect of the lower dose of fish oil, which does better than the higher dose. We would like to share with the authors the following comments:

First, there is no control group and the outcome might be purely due to a placebo effect, as this effect is high in any kind of trials in patients with knee OA. ${ }^{3}$

It is possible that the authors 'select' a population of patients with mild knee OA. It was not made clear which kind of OA was selected: patellar or tibiofemoral knee OA? The inclusion criteria for pain are unusually low: 'any patient with a pain superior of $20 \mathrm{~mm}$ on a $100 \mathrm{~mm}$ VAS'. Actually, the Western Ontario and McMaster Universities (WOMAC) pain subscore at baseline (0-50) is low in both groups (15 in the low-dose group and 13 in the high-dose group). Thus, it is possible that part of the patients recruited in this trial present with a level of pain $<30 \mathrm{~mm}$, meaning an acceptable level of pain at baseline, too low to observe any difference over time. ${ }^{45}$

In the same line, more than $70 \%$ of the participants had no change in the cartilage volume or bone marrow lesion (BML) size over time during the 2 years of follow-up. Change in knee cartilage volume is frequently used as a proxy for change in knee joint space width over time. A recent longitudinal study in a Tasmanian population shows that cartilage volume decreased weakly but significantly over time, though it was not related with joint space width. ${ }^{6}$ Otherwise the authors do not correlate the clinical changes with presence and evolution of BML. A recent work demonstrated a link between BMLs present on both T1-weighted and T2-weighted and not only on T2-weighted MRI sequences, and increased medial tibial cartilage loss and incident knee pain in patients with knee OA over 2 years of follow-up. ${ }^{7}$ Taking clinical and MRI data together in this trial, it might suggest a bias in selection of patients with low symptomatic status and a non-progressing disease.

The differences between the two groups are small at 1 year and even at 2 years. This suggests that those differences could be clinically non-significant even if statistically significant. ${ }^{4}$ Indeed, the authors did not observe a decrease in consumption of paracetamol between groups.

At the end of the day, the main query concerned fish oil utility in our daily practice. Actually, patients with OA are very keen on any kind of dietary supplement to manage their disease. In a recent survey on patient's expectations, one of the selected item was 'Informs me about all the treatment modalities including complementary medicine'.

In other words, should we recommend fish oil over 18 months in our patients? At least, we should not recommend high dose of fish oil.... On the other hand, the adverse events in terms of gastrointestinal tolerance, though non-serious, were present in more than $60 \%$ of the patients... A small benefit for the high frequency of side effects.

\section{Chevalier, F Eymard}

Department of Rheumatology, UPEC Paris XII University, Creteil, France

Correspondence to Professor X Chevalier, Department of Rheumatology, UPEC Paris XII University, Hopital Henri Mondor, Bd De Lattre de Tassigny, Creteil 94010, France; xavier.chevalier@aphp.fr

Contributors Alls contributors certify that they have collaborated, read and reviewed this correspondence and have no competing interest.

Competing interests None declared.

Provenance and peer review Not commissioned; internally peer reviewed.

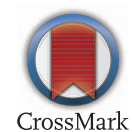

To cite Chevalier X, Eymard F. Ann Rheum Dis 2016;75:e41.

Received 18 February 2016

Accepted 20 February 2016

Published Online First 10 March 2016

Ann Rheum Dis 2016;75:e41. doi:10.1136/annrheumdis-2016-209399

\section{REFERENCES}

1 Hill CL, March LM, Aitken D, et al. Fish oil in knee osteoarthritis: a randomised clinical trial of low dose versus high dose. Ann Rheum Dis 2016;75:23-9.

2 Felson DT, Bischoff-Ferrari HA. Dietary fatty acids for the treatment of OA, including fish oil. Ann Rheum Dis 2016:75:1-2

3 Bannuru RR, McAlindon TE, Sullivan MC, et al. Effectiveness and Implications of Alternative Placebo Treatments: A Systematic Review and Network Meta-analysis of Osteoarthritis Trials. Ann Intern Med 2015;163:365-72.

4 Pham T, Tubach F. Patient acceptable symptomatic state (PASS). Joint Bone Spine 2009;76:321-3.

5 Tubach F, Giraudeau B, Ravaud P. The variability in minimal clinically important difference and patient acceptable symptomatic state values did not have an impact on treatment effect estimates. I Clin Epidemiol 2009;62:725-8.

6 Hall J, Laslett LL, Martel-Pelletier J, et al. Change in knee structure and change in tibiofemoral joint space width: a five year longitudinal population-based study. BMC Musculoskelet Disord 2016;17:25.

7 Wluka AE, Teichtahl AJ, Maulana R, et al. Bone marrow lesions can be subtyped into groups with different clinical outcomes using two magnetic resonance imaging (MRI) sequences. Arthritis Res Ther 2015;17:270.

8 Benhamou M, Boutron I, Dalichampt $\mathrm{M}$, et al. Elaboration and validation of a questionnaire assessing patient expectations about management of knee osteoarthritis by their physicians: the Knee Osteoarthritis Expectations Questionnaire. Ann Rheum Dis 2013:72:552-9. 